\title{
Macrofauna invertebrada edáfica em cultivo de mandioca sob sistemas de cobertura do solo
}

\author{
Rogério Ferreira da Silva( ${ }^{(1)}$, Michely Tomazi(2), Carmen Regina Pezarico(3), Adriana Maria de Aquino(4) $^{(4)}$ \\ e Fábio Martins Mercante ${ }^{(1)}$
}

\begin{abstract}
(1)Embrapa Agropecuária Oeste, Caixa Postal 661, CEP 79804-970 Dourados, MS. E-mail: rogerio@cpao.embrapa.br,_mercante@cpao.embrapa.br (2)Universidade Federal do Rio Grande do Sul, Caixa Postal 15100, CEP 90001-970 Porto Alegre, RS. E-mail: mitomazi@yahoo.com.br (3)Agência de Desenvolvimento Agrário e Extensão Rural de Mato Grosso do Sul, Rua Tiradentes, 798, CEP 79900-000 Ponta Porã, MS. E-mail: pezarico@gmail.com (4)Embrapa Agrobiologia, CEP 23851-970 Seropédica, RJ. E-mail: adriana@cnpab.embrapa.br
\end{abstract}

\begin{abstract}
Resumo - O objetivo deste trabalho foi avaliar o efeito do cultivo da mandioca em diferentes sistemas de cobertura do solo na densidade e diversidade da comunidade da macrofauna de invertebrados edáfica. $\mathrm{O}$ trabalho foi conduzido no Município de Glória de Dourados, MS, num Argissolo Vermelho, sob sistema convencional (SC), plantio direto sobre palhada de mucuna (PDMu), sorgo (PDSo) e milheto (PDMi), além de sistema com vegetação nativa $(\mathrm{VN})$, como referencial para comparação. As avaliações foram realizadas em quatro épocas distintas: abril/2003 (antes do plantio), novembro/2003 (6 meses após o plantio), abril/2004 (11 meses após o plantio) e novembro/2004 (18 meses após o plantio). Houve efeito da interação entre os sistemas avaliados e as épocas de amostragens sobre a densidade, riqueza e diversidade da macrofauna invertebrada do solo. Entre os grupos da macrofauna invertebrada do solo, cupins, formigas e coleópteros (imaturo e adulto) foram predominantes no ambiente estudado. $\mathrm{O}$ uso de plantas de cobertura no pré-cultivo de mandioca no sistema plantio direto proporcionou condições para a recomposição da comunidade de macrofauna invertebrada do solo, o que indica que as espécies utilizadas, mucuna, sorgo e milheto, representam alternativas promissoras para melhor manejo dessa cultura.
\end{abstract}

Termos para indexação: Manihot esculenta, plantio direto, densidade, diversidade.

\section{Edaphic invertebrate macrofauna in cassava cultivation under vegetable cover crops}

\begin{abstract}
The objective of this work was to evaluate the effect of cassava cultivation under different vegetable cover crops according to the density and diversity of soil invertebrate macrofauna. Field experiment was carried out at Glória de Dourados, Mato Grosso do Sul State, Brazil, on an Oxisol, under conventional drilling (SC), no-tillage system under Stizolobium cinereum (PDMu), Sorghum bicolor (PDSo) and Pennisetum glaucum (PDMi) mulching, with comparison of native vegetation system (VN). Evaluations were performed in April/2003 (before sowing), November/2003 (6 months after sowing), April/2004 (11 months after sowing) and November/ 2004 (18 months after sowing). Significant effect of the interaction between the evaluated systems and sampling dates in relation to density and diversity values of soil invertebrate macrofauna was observed. The termites, ants and Coleoptera (immature and adult) groups were predominant in this study. The use of cover crops plants in the cassava pre-sowing, under no-tillage, provided conditions for the reestablishment of soil invertebrate macrofauna community, indicating that Stizolobium cinereum, Sorghum bicolor and Pennisetum glaucum are promising alternatives for a better management.
\end{abstract}

Index terms: Manihot esculenta, no-tillage, density, diversity.

\section{Introdução}

A cultura da mandioca (Manihot esculenta Crantz) se destaca como fonte de energia na alimentação humana e animal, na indústria de derivados, e, conseqüentemente, como geradora de emprego e renda. A cultura ocupou, na safra brasileira de 2005,
1.905.361 ha, com uma produção de 26.405.130 t, que resultou em um rendimento médio nacional, de 13,86 tha-1 (Mandioca..., 2006), posicionando-a em segundo lugar na produção mundial, e entre as principais explorações agrícolas do País.

No Estado de Mato Grosso do Sul, a área plantada com mandioca aumentou de 27.658 ha em 1998, para 
40.941 ha em 2005 (Mandioca..., 2006). Esse crescimento foi acompanhado pelo desenvolvimento da indústria, aumentando a geração de emprego e renda neste setor, que é explorado principalmente pela população de baixa renda (Cardoso \& Leal, 2005).

Nessa cultura, o preparo do solo é tradicionalmente realizado com uma aração e duas gradagens, o que pode provocar a degradação da sua qualidade, como a erosão, encrostamento superficial, oxidação da matéria orgânica, redução da atividade biológica e, conseqüentemente, a perda da sua capacidade produtiva com cultivos sucessivos. A adoção de sistemas de preparo com mínimo revolvimento do solo e uso de plantas de cobertura, além de oferecerem as condições favoráveis ao crescimento e desenvolvimento da cultura, fundamentam a sustentabilidade dos sistemas de produção (Cavalieri et al., 2006).

Vários trabalhos enfatizam a importância da rotação de culturas, com o uso de plantas de cobertura do solo, no cultivo das espécies vegetais, já que, além de proteger o solo contra os agentes erosivos, a rotação contribui para melhoria das propriedades físicas, químicas e biológicas do solo (Dao, 1996; Oliveira et al., 2002). A adoção desses sistemas, que mantêm na superfície do solo os resíduos de culturas de cobertura, tem sido preconizada por constituir uma alternativa tecnicamente viável para a cultura da mandioca (Oliveira et al., 2001). Embora essa técnica venha sendo utilizada pelos produtores de Mato Grosso do Sul, principalmente quando o solo é arenoso ou muito arenoso, há poucas informações sobre sua eficiência na manutenção da qualidade do solo (Souza \& Souza, 2002), principalmente em relação aos processos biológicos em que se destaca a fauna edáfica, que habita o complexo serapilheirasolo, com importante atuação na ciclagem de nutrientes e estrutura física do solo (Barros et al., 2001; Lavelle \& Spain, 2001).

Os invertebrados com diâmetro corporal acima de $2 \mathrm{~mm}$ constituem a macrofauna, à qual pertencem os grupos de minhocas, coleópteros em estado larval e adulto, centopéias, cupins, formigas, diplópodes, isópodes e aracnídeos (Lavelle et al., 1997; Wolters, 2000; Lavelle \& Spain, 2001). Esses invertebrados são fundamentais ao funcionamento do ecossistema, pois ocupam todos os níveis tróficos na cadeia alimentar do solo e afetam a produção primária de maneira direta e indireta.

Vários trabalhos evidenciaram que o tipo de manejo do solo acarreta inúmeras modificações na estrutura da comunidade de macroinvertebrados, em diferentes graus de intensidade em virtude de mudanças de habitat, fornecimento de alimento e criação de microclimas (Decäens et al., 2003; Merlim et al., 2005). Essas modificações geralmente ocorrem na diversidade e densidade populacionais, características que têm sido utilizadas como potencial bioindicador da qualidade do solo (Coleman \& Hendrix, 2000; Lavelle \& Spain, 2001; Silva et al., 2006a, 2006b). O objetivo deste trabalho foi avaliar os efeitos do cultivo da mandioca em diferentes sistemas de cobertura do solo na densidade e diversidade da comunidade da macrofauna invertebrada edáfica.

\section{Material e Métodos}

O trabalho foi conduzido entre 2002 e 2004 no campo experimental da Prefeitura Municipal de Glória de Dourados, MS ( $22^{\circ} 22^{\prime} \mathrm{S}$ e $54^{\circ} 30^{\prime} \mathrm{W}, 400 \mathrm{~m}$ de altitude), em solo classificado como Argissolo Vermelho, textura arenosa. O clima de ocorrência, segundo a classificação de Köppen, é do tipo Aw, com estação quente e chuvosa no verão e moderadamente seca no inverno. Os dados agroclimáticos mensais de precipitação pluvial $(\mathrm{mm})$ e temperatura $\left({ }^{\circ} \mathrm{C}\right)$ durante o período de estudo encontram-se na Figura 1.

Antes do estabelecimento da cultura da mandioca, a área experimental estava sendo utilizada com pastagem de Brachiaria brizantha (Hochst. ex A. Rich.) Stapf. Na safra 2001/2002, foi estabelecida a cultura do milho em sistema convencional, visando à homogeneização da área.

Este experimento iniciou-se em outubro de 2002, com a divisão dessa área em quatro talhões de $1.800 \mathrm{~m}^{2}$, nos quais foram efetuadas a semeadura de mucuna-cinza

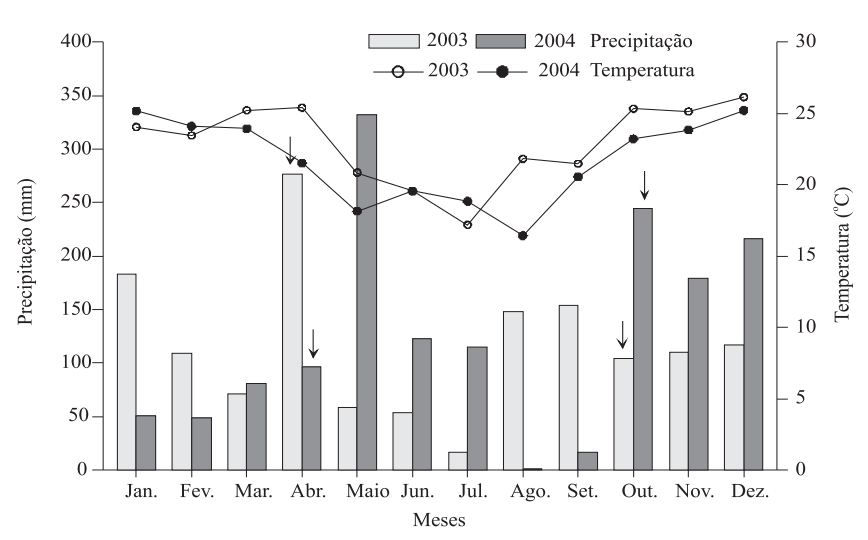

Figura 1. Precipitação pluvial e temperatura média mensal, no Município de Glória de Dourados, MS, referentes aos anos de 2003 e 2004. As setas indicam as épocas de avaliação. 
(Stizolobium cinereum Piper e Tracy), sorgo-granífero (Sorghum bicolor (L.) Moench) e milheto (Pennisetum americanum L.), sob preparo convencional, com aração e gradagens. No quarto talhão, a área foi deixada em pousio para posterior preparo com aração e gradagens ( sistema convencional), que serviu como padrão comparativo. Realizou-se, também amostragem de solo numa área sob vegetação nativa (Cerrado), adjacente às parcelas experimentais, que serviram como padrão para comparação.

Nas áreas em que foram cultivadas as diferentes espécies vegetais, foram aplicados $495 \mathrm{~kg} \mathrm{ha}^{-1}$ de fertilizante mineral NPK (0-20-20). Na floração plena, essas culturas passaram por um processo de rolagem utilizando um rolo-faca e foram dessecadas com glyphosate e 2,4-D na dosagem recomendada. Após a dessecação, em maio de 2003, efetuou-se o plantio da mandioca, variedade Fécula Branca, em fileira simples com espaçamento de $0,9 \mathrm{~m}$ entre fileiras e $0,7 \mathrm{~m}$ entre plantas, com aplicação de $456 \mathrm{~kg} \mathrm{ha}^{-1}$ de fertilizante mineral NPK (4-20-20). A colheita foi realizada em novembro de 2004, aos 18 meses após o plantio.

As amostragens de solo para extração da fauna foram realizadas em quatro épocas distintas: antes do plantio da mandioca (abril/2003), que ocorreu logo após a dessecação das espécies de cobertura nas parcelas sob plantio direto; 6 meses após o plantio da mandioca (novembro/2003); 11 meses após o plantio da mandioca (abril/2004); e 18 meses após o plantio da mandioca (novembro/2004). Foram amostrados, em cada sistema, cinco monólitos de solo de $0,25 \times 0,25 \mathrm{~m}$ de largura e $0,30 \mathrm{~m}$ de profundidade, subdivididos em camadas de $0,10 \mathrm{~m}$, ao longo de um transecto, eqüidistantes $5 \mathrm{~m}$, de acordo com Anderson \& Ingram (1993), e a macrofauna extraída foi conservada em solução de álcool 70\%. Com auxílio de lupa binocular, realizou-se a identificação e contagem dos organismos, em nível de grandes grupos taxonômicos, atuantes no conjunto serapilheira-solo. Após a extração da macrofauna, o solo foi caracterizado quimicamente (Tabela 1), de acordo com Claessen (1997).

O delineamento experimental adotado foi o de blocos casualizados em um esquema de parcelas subdivididas, com cinco repetições. As parcelas principais, com cerca de $350 \mathrm{~m}^{2}$, foram os sistemas de preparo do solo: sistema convencional (SC), plantio direto sobre resíduos culturais de mucuna (PDMu), sorgo (PDSo) e milheto (PDMi), além de sistema com vegetação nativa $(\mathrm{VN})$ e as subparcelas, as épocas de avaliação (1- época - abril/ 2003, 2a época - novembro/2003, 3a época - abril/2003 e 4a época - novembro/2004).

A comunidade da macrofauna invertebrada edáfica foi avaliada por meio da densidade (indivíduos por $\mathrm{m}^{2}$ ), riqueza ( $\mathrm{n}^{\mathrm{o}}$ de grupos) e diversidade (índice de Shannon). O índice de diversidade de Shannon foi obtido pela relação $\left(\mathrm{H}=-\sum_{\mathrm{i}-1}\right.$ pi $\left.1 \mathrm{n} \mathrm{pi}\right)$, em que: $\mathrm{pi}=$ ni/N; ni é a densidade de cada grupo; $\mathrm{N}$ é o número total de grupos, conforme Magurran (1988).

Os dados obtidos (x), por sua heterogeneidade, foram transformados em $(x+0,5)^{1 / 2}$ e depois submetidos à análise de variância (teste $\mathrm{F}$ ); as médias foram comparadas pelo teste de Duncan, a $5 \%$ de probabilidade. Efetuou-se o teste de coeficiente de correlação de Pearson com dados referentes à densidade total e riqueza de grupos da macrofauna, de acordo com o aporte de resíduos culturais e conteúdo de matéria orgânica do solo. Além disso, os dados foram submetidos à análise de agrupamento ("cluster analysis"), adotando-se o método do vizinho mais distante ("complete linkage") a partir da distância euclidiana, a fim de descrever a similaridade entre os sistemas. As análises estatísticas foram processadas por meio de software Statistica (versão 5.0, StatSoft).

\section{Resultados e Discussão}

Houve efeito significativo $(\mathrm{p}<0,05)$ da interação entre os sistemas avaliados e as épocas de amostragens sobre

Tabela 1. Características químicas do solo sob sistema convencional (SC), plantio direto (PD) sobre palhada de mucuna (Mu), sorgo (So) e milheto (Mi) e vegetação nativa (VN). Valores médios de quatro épocas de avaliação.

\begin{tabular}{lcccccc}
\hline Uso do solo & $\mathrm{pH}\left(\mathrm{H}_{2} \mathrm{O}\right)$ & $\begin{array}{c}\mathrm{P} \\
\left(\mathrm{mg} \mathrm{dm}^{-3}\right)\end{array}$ & $\mathrm{K}$ & $\mathrm{Ca}$ & $\begin{array}{c}\mathrm{Mg} \\
\mathrm{MO} \\
\left(\mathrm{g} \mathrm{kg}^{-1}\right)\end{array}$ \\
\hline SC & 5,2 & 6,6 & 0,15 & 0,60 & 0,23 & 0,28 \\
PDMu & 5,1 & 11,5 & 0,13 & 0,61 & 0,32 & 0,35 \\
PDSo & 5,1 & 13,3 & 0,19 & 0,75 & 0,30 & 0,3 \\
PDMi & 5,2 & 10,6 & 0,20 & 0,65 & 0,39 & 0,3 \\
VN & 5,0 & 1,9 & 0,08 & 0,44 & 0,34 & 0,48 \\
\hline
\end{tabular}


os valores de densidade, riqueza e diversidade da macrofauna invertebrada do solo (Tabela 2). Antes do plantio da mandioca (abril/2003), observou-se que os sistemas PDSo, PDMi e VN apresentaram os valores de densidade semelhantes (média de 800 ind $\mathrm{m}^{-2}$ ) e significativamente superiores ao SC e PDMu (54 e 324 ind $\mathrm{m}^{-2}$, respectivamente) (Tabela 2 ). O SC apresentou também a menor riqueza e diversidade em relação aos demais sistemas avaliados. Provavelmente, nesse sistema, a fauna foi influenciada pelas modificações impostas pela aração e gradagens, como a destruição do habitat, exposição aos predadores, eliminação do alimento disponível e condições edafoclimáticas desfavoráveis, como temperatura e umidade (Decaëns et al., 2003; Silva et al., 2006a).

Nas demais épocas de avaliação (novembro/2003, abril/2004 e novembro/2004), os valores de densidade, riqueza e diversidade não diferiram significativamente entre os sistemas cultivados (Tabela 2), exceto a riqueza no PDSo, na avaliação de abril/2004, que apresentou valores superiores aos demais sistemas cultivados. Isso indica que o uso de plantas de cobertura do solo, como mucuna, sorgo e milheto, no cultivo de mandioca tem efeito positivo apenas no período de estabelecimento da cultura.
Na avaliação de novembro/2003, referente ao estádio inicial de desenvolvimento da cultura da mandioca (seis meses), houve redução na riqueza de grupos da comunidade da macrofauna invertebrada do solo, em relação à primeira avaliação (abril/2003), nos sistemas com plantas de cobertura. Essa variação pode estar relacionada às características da cultura da mandioca, que não proporcionam boa proteção ao solo em seu estádio inicial de desenvolvimento, em virtude do baixo índice de área foliar, associado ao amplo espaçamento utilizado $(0,7 \times 0,9 \mathrm{~m})$.

Outro fator que pode ter contribuído negativamente na manutenção da riqueza da macrofauna edáfica foi a redução na quantidade de resíduos culturais das plantas de cobertura, em relação à primeira avaliação. Estes resíduos vegetais constituem a principal fonte de alimento e habitat para grande parte das espécies de invertebrados que habitam o solo (Marasas et al., 2001; Barros et al., 2003; Benito et al., 2004; Silva et al., 2006a).

A densidade e a riqueza de grupos da comunidade de macrofauna edáfica apresentaram correlação positiva e significativa $(\mathrm{p}<0,05)$ entre os diferentes tipos de resíduos culturais $(r=0,55$ e $\mathrm{r}=0,79$, respectivamente) e o conteúdo da matéria orgânica $(r=0,59$ e $r=0,67$, respectivamente). Isso indica a influência da matéria

Tabela 2. Valores médios de densidade (indivíduos por $\mathrm{m}^{2}$ ), com respectivos erros-padrões; riqueza (n⿳⺈⿴囗十一 de grupos taxonômicos) e diversidade da comunidade de macrofauna invertebrada do solo sob diferentes sistemas de cultivo de mandioca ${ }^{(1)}$.

\begin{tabular}{|c|c|c|c|c|c|}
\hline \multirow[t]{2}{*}{ Sistemas } & & \multicolumn{3}{|c|}{ Épocas de avaliação } & \multirow[t]{2}{*}{ Médias } \\
\hline & Abril/2003 & Novembro/2003 & Abril/2004 & Novembro/2004 & \\
\hline \multicolumn{6}{|c|}{ Densidade } \\
\hline $\mathrm{SC}$ & $54 \pm 13 \mathrm{Bc}$ & $61 \pm 9 \mathrm{Bb}$ & $294 \pm 104 \mathrm{Ab}$ & $217 \pm 107 \mathrm{Aa}$ & 157 \\
\hline PDMu & $323 \pm 116 \mathrm{ABb}$ & $45 \pm 14 \mathrm{Bb}$ & $160 \pm 88 \mathrm{Bb}$ & $778 \pm 317 \mathrm{Aa}$ & 326 \\
\hline PDSo & $912 \pm 313 \mathrm{Aa}$ & $106 \pm 34 \mathrm{Bb}$ & $579 \pm 256 \mathrm{ABab}$ & $570 \pm 247 \mathrm{ABa}$ & 542 \\
\hline PDMi & $822 \pm 216 \mathrm{Aa}$ & $211 \pm 103 \mathrm{Bb}$ & $122 \pm 66 \mathrm{Bb}$ & $880 \pm 375 \mathrm{Aa}$ & 509 \\
\hline $\mathrm{VN}$ & $838 \pm 168 \mathrm{Ba}$ & $2154 \pm 236 \mathrm{Aa}$ & $1446 \pm 219 \mathrm{Aa}$ & $621 \pm 176 \mathrm{Ba}$ & 1.265 \\
\hline \multicolumn{6}{|c|}{ Riqueza } \\
\hline $\mathrm{SC}$ & 2(4)Bc & 2(4)Bb & $3(6) \mathrm{ABb}$ & $4(9) \mathrm{Ab}$ & 3 \\
\hline $\mathrm{PDMu}$ & $5(9) \mathrm{Ab}$ & 2(4)Bb & $2(5) \mathrm{Bb}$ & 5(10)Aab & 4 \\
\hline PDSo & $6(10) \mathrm{Aab}$ & $3(6) \mathrm{Bb}$ & 6(8)Aa & $6(8) \mathrm{Aab}$ & 5 \\
\hline PDMi & 7(11)Aa & $3(6) \mathrm{Bb}$ & $3(8) \mathrm{Bb}$ & 6(9)Aab & 5 \\
\hline $\mathrm{VN}$ & 6(10)Aab & $6(10) \mathrm{Aa}$ & 6(9)Aa & $8(11) \mathrm{Aa}$ & 7 \\
\hline \multicolumn{6}{|c|}{ Diversidade } \\
\hline $\mathrm{SC}$ & $0,14 \mathrm{Bb}$ & $0,36 \mathrm{ABa}$ & $0,30 \mathrm{ABa}$ & $0,51 \mathrm{Aa}$ & 0,33 \\
\hline $\mathrm{PDMu}$ & $0,52 \mathrm{Aa}$ & $0,23 \mathrm{Ba}$ & $0,26 \mathrm{ABa}$ & $0,51 \mathrm{Aa}$ & 0,38 \\
\hline PDSo & $0,56 \mathrm{Aa}$ & $0,41 \mathrm{Aa}$ & $0,57 \mathrm{Aa}$ & $0,42 \mathrm{Aa}$ & 0,49 \\
\hline PDMi & $0,62 \mathrm{Aa}$ & $0,29 \mathrm{Aa}$ & $0,42 \mathrm{Aa}$ & $0,46 \mathrm{Aa}$ & 0,44 \\
\hline $\mathrm{VN}$ & $0,53 \mathrm{Ba}$ & $0,34 \mathrm{Ca}$ & $0,47 \mathrm{BCa}$ & $0,70 \mathrm{Aa}$ & 0,51 \\
\hline
\end{tabular}

${ }^{(1)}$ Médias com letras diferentes, minúsculas nas colunas e maiúsculas nas linhas, contrastam pelo teste de Duncan, a 5\% de probabilidade; para a análise estatística, os valores foram transformados em $(x+0,5)^{1 / 2}$; os dados entre parênteses de riqueza de grupos referem-se à riqueza total acumulada, considerando-se as cinco repetições; SC: sistema convencional; PD: plantio direto sobre resíduos de mucuna (Mu), sorgo (So) e milheto (Mi); VN: vegetação nativa. 
orgânica na comunidade da fauna do solo e sua ação na fragmentação e umificação da matéria orgânica. A presença de cobertura permanente no solo contribui para o aumento da disponibilidade de energia associada à existência de novos habitats favoráveis à colonização dos organismos invertebrados, o que pode beneficiar a sustentabilidade ecológica dos sistemas de produção.

As práticas de manejo do solo, com utilização, principalmente, de sistemas com coberturas de resíduos, influenciaram a densidade e riqueza de grupos da comunidade de macrofauna edáfica, durante o desenvolvimento da cultura da mandioca (Tabela 2). A dinâmica da comunidade da macrofauna seguiu a mesma tendência de variação nos sistemas cultivados; reduziu ou aumentou de acordo com a época de avaliação. Esta oscilação na densidade e riqueza da macrofauna edáfica parece relacionar-se com a variação de temperatura e umidade nos sistemas. Em razão da íntima associação com os processos que ocorrem no compartimento serapilheira-solo e a sua grande sensibilidade a fatores bióticos e abióticos, a diversidade da comunidade da macrofauna do solo reflete o padrão de funcionamento do ecossistema (Odun, 1988).

Os cupins (Isoptera) representaram, em média, 40,5\% do total de organismos coletados, seguidos das formigas (Formicidae), dos coleópteros (Coleoptera) imaturos e adultos (Tabela 3). O tipo de manejo do solo pode influenciar a abundância de indivíduos e a predominância de grupos em determinado sistema (Marasas et al., 2001; Lavelle et al., 2001; Barros et al., 2003). Entre os sistemas avaliados, observou-se a predominância das formigas (Formicidae) no SC $(34,8 \%)$, PDMu $(53,1 \%)$ e PDMi $(57,3 \%)$, e dos cupins (Isoptera) no PDSo $(44,3 \%)$ e VN $(59,1 \%)$. Resultados similares foram observados por Silva et al. (2006a), em sistema plantio direto para culturas anuais com diferentes coberturas vegetais, em um Latossolo Vermelho distroférrico típico. Estes organismos têm sido descritos como engenheiros ecológicos do solo, pois as estruturas biogênicas que produzem são importantes no sistema, representando sítios em que ocorrem processos fundamentais, como a estimulação da atividade microbiana, a formação de estrutura de solo e a dinâmica da matéria orgânica (Jones et al., 1994; Lavelle \& Spain, 2001).

Outro grupo com importância destacada foi Coleoptera (imaturo e adulto) no PDMu (Tabela 3), provavelmente em razão de a maioria dos organismos encontrados ser saprófago e encontrar nos resíduos da mucuna alimento com baixa relação $\mathrm{C} / \mathrm{N}$.

De acordo com a análise de agrupamento, foi possível a formação de dois grupos distintos, um formado pelo SC (em todas as épocas de avaliação), PDMu, PDSo e PDMi (aos 6 a 11 meses após o plantio da mandioca) e outro formado pela VN (em todas as épocas de avaliação), PDMu, PDSo e PDMi (antes do plantio da mandioca e aos 18 meses após o plantio). Esses dois grupos não apresentaram nenhuma similaridade entre si, uma vez que a sua distância de ligação foi de $100 \%$ (Figura 2).

O primeiro grupo engloba a ligação com distância inferior a $12 \%$ entre os sistemas avaliados, o que permite inferir que a similaridade entre eles é de $88 \%$. Isso demonstra que o cultivo de mandioca sobre resíduos de mucuna, sorgo e milheto, do 6o ao 11으 mês após o plantio, apresenta características da estrutura

Tabela 3. Distribuição relativa (\%), média de quatro épocas de avaliação, dos grupos da macrofauna invertebrada do solo sob sistema convencional (SC), plantio direto (PD) sobre resíduos de mucuna (Mu), sorgo (So) e milheto (Mi), além da vegetação nativa (VN).

\begin{tabular}{|c|c|c|c|c|c|c|}
\hline Grupos & $\mathrm{SC}$ & $\mathrm{PDMu}$ & PDSo & PDMi & VN & Média \\
\hline Oligochaeta & 0,5 & 0,0 & 0,9 & 0,8 & 0,5 & 0,5 \\
\hline Chilopoda & 1,5 & 0,0 & 1,0 & 1,5 & 0,5 & 0,7 \\
\hline Heteroptera & 1,5 & 2,0 & 2,1 & 2,6 & 0,4 & 1,4 \\
\hline Araneae & 1,5 & 1,2 & 1,5 & 2,0 & 2,1 & 1,8 \\
\hline Hymenoptera $^{(1)}$ & 9,2 & 1,5 & 4,6 & 1,5 & 0,0 & 1,9 \\
\hline Coleoptera & 5,7 & 9,4 & 5,2 & 8,7 & 2,4 & 5,2 \\
\hline Larva de Coleoptera & 15,9 & 18,2 & 13,9 & 9,3 & 10,2 & 12,0 \\
\hline Formicidae & 34,9 & 53,1 & 24,8 & 57,3 & 20,5 & 32,8 \\
\hline Isoptera & 28,2 & 10,3 & 44,3 & 14,2 & 59,1 & 40,5 \\
\hline Outros & 1,1 & 4,3 & 1,7 & 2,1 & 4,3 & 3,2 \\
\hline
\end{tabular}

${ }^{(1)}$ Excluindo-se Formicidae. 
da macrofauna invertebrada do solo semelhante ao sistema convencional. Este fato pode estar relacionado, além das características da cultura da mandioca, com pouca proteção do solo em seu estádio inicial de desenvolvimento, à redução na quantidade de resíduos culturais das plantas de cobertura, principal fonte de alimento e habitat para grande parte dos organismos invertebrados do solo.

No outro grupo, observou-se que os sistemas PDMu, PDSo e PDMi (primeira e última avaliações) apresentaram 52,5\% de similaridade com VN (em todas as épocas de avaliação). A formação desse grupo, provavelmente, ocorreu em virtude do efeito marcante dos resíduos culturais das plantas de cobertura (mucuna, sorgo e milheto) sobre a macrofauna invertebrada do solo, que desaparece no decorrer do desenvolvimento da cultura da mandioca. Além disso, constatou-se que, no final do ciclo da cultura da mandioca (última avaliação), esses sistemas de manejo apresentam características da macrofauna edáfica próximas às verificadas na $\mathrm{VN}$ e indicam que o uso de plantas de coberturas no cultivo de mandioca influencia positivamente a qualidade do solo, o que representa uma alternativa promissora para esta cultura.

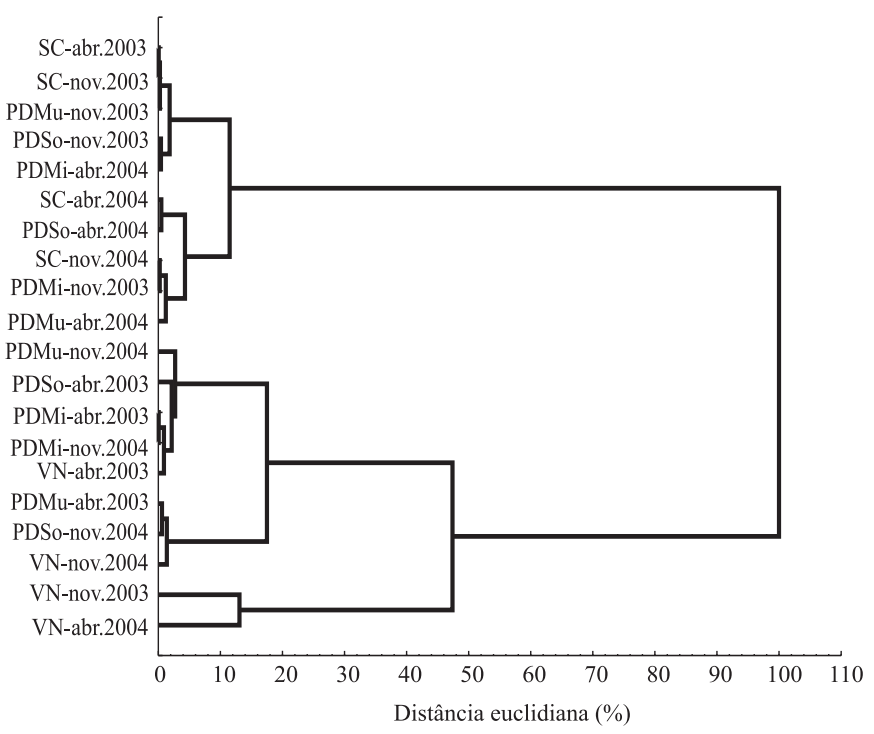

Figura 2. Dendrograma de similaridade da comunidade de macrofauna edáfica entre os sistemas de manejo de solo, em diferentes épocas, com bases nas distâncias euclidianas. $\mathrm{SC}$ : sistema convencional; PD: plantio direto sobre resíduos de mucuna $(\mathrm{Mu})$, sorgo (So) e milheto (Mi); VN: vegetação nativa.

\section{Conclusões}

1. A comunidade da macrofauna do solo responde aos impactos causados pelo manejo e constitui-se num bom indicador para avaliação da qualidade dos solos submetidos a diferentes sistemas de manejo.

2. O uso de plantas de cobertura no pré-cultivo de mandioca no sistema plantio direto proporciona condições para a recomposição da comunidade de macrofauna invertebrada do solo.

3. As espécies utilizadas, mucuna, sorgo e milheto, representam alternativas promissoras para bom manejo da cultura da mandioca.

\section{Agradecimentos}

Ao CNPq e à Fundação de Apoio ao Desenvolvimento do Ensino, Ciência e Tecnologia do Estado de Mato Grosso do Sul (Fundect), pelo suporte financeiro.

\section{Referências}

ANDERSON, J.M.; INGRAM, J.S.I. (Ed.). Tropical soil biological and fertility: a handbook of methods. $2^{\text {nd }}$ ed. Wallingford: CAB International, 1993. 221p.

BARROS, E.; CURMI, P.; HALlAIRE, V.; CHAUVEL, A.; LAVELLE, P. The role of macrofauna in the transformation and reversibility of soil structure of an oxisol in the process of forest to pasture conversion. Geoderma, v.100, p.193-213, 2001.

BARROS, E.; NEVES, A.; BLANCHAR, E.; FERNANDES, E.C.M.; WANDELLI, E.; LAVELLE, P. Development of the soil macrofauna community under silvopastoral and agrosilvicultural systems in Amazônia. Pedobiologia, v.47, p.1-7, 2003.

BENITO, N.P.; BROSSARD, M.; PASINI, A.; GUIMARÃES, M.F.; BOBILLIER, B. Transformations of soil macroinvertebrate populations after native vegetation conversion to pasture cultivation (Brazilian Cerrado). European Journal of Soil Biology, v.40, p.147154, 2004.

CARDOSO, C.E.L.; LEAL, M.S. Algumas mudanças na cadeia de mandioca e suas implicações. Revista Brasileira de Mandioca, v.18, p.33-44, 2005.

CAVALIERI, K.M.V.; TORMENA, C.A.; VIDIGAL FILHO, P.S.; GONÇALVES, A.C.A.; COSTA, A.C.S. Efeitos de sistemas de preparo nas propriedades físicas de um Latossolo Vermelho Distrófico. Revista Brasileira de Ciência do Solo, v.30, p.137147, 2006.

CLAESSEN, M.E.C. (Org.). Manual de métodos de análise de solo. 2.ed. rev. atual. Rio de Janeiro: Embrapa-CNPS, 1997. 212p. (Embrapa-CNPS.Documentos, 1).

COLEMAN, D.C.; HENDRIX, P.F. Invertebrates as webmasters in ecossystems. London: CABI, 2000. 336p. 
DAO, T.H. Tillage system and crop residue effects on surface compaction of a paleusoll. Agronomy Journal, v.88, p.141-148, 1996.

DECAËNS, T.; JIMÉNEZ, J.J.; ESCOBAR, G.; RIPPSTEIN, G.; SCHNEIDMADL, J.; SANZ, J.I.; HOYOS, P.; THOMAS, R.J. Impacto del uso de la tierra en la macrofauna del suelo de los Llanos Orientales de Colombia. In: JIMÉNEZ, J.J.; THOMAS, R.J. (Ed.). El arado natural: las comunidades de macroinvertebrados del suelo en las savanas neotropicales de Colombia. Cali: Centro Internacional de Agricultura Tropical, 2003. p.21-45. (CIAT. Publicación, 336).

JONES, C.G.; LAWTON, J.H.; SHACHAK, M. Organisms as ecosystem engineers. Oikos, v.69, p.373-386, 1994.

LAVELLE, P.; BARROS, E.; BLANCHART, E.; BROWN, G.; DESJARDINS, T.; MARIANI, L.; ROSSI, J.P. SOM management in the tropics: why feeding the soil macrofauna? Nutrient Cycling in Agroecosystems, v.61, p.53-61, 2001.

LAVELLE, P.; BIGNELL, D.; LEPAGE, M.; WOLTERS, V.; ROGER, P.; INESON, P.; HEAL, O.W.; DHILLION, S. Soil function in a changing world: the role of invertebrate ecosystem engineers. European Journal of Soil Biology, v.33, p.159-193, 1997.

LAVELLE, P.; SPAIN, A.V. Soil ecology. Dordrecht: Kluwer Academic, 2001. 654p.

MAGURRAN, A.E. Ecological diversity and its measurement. New Jersey: Princenton Univ. Press, 1988. 179p.

MANDIOCA: produção brasileira. Agrianual 2006: Anuário da Agricultura Brasileira, São Paulo, p.360, [out. 2005].

MARASAS, M.E.; SARANDÓN, S.J.; CICCHINO, A.C. Changes in soil arthropod functional group in a wheat crop under convencional and no tillage systems in Argentina. Applied Soil Ecology, v.18, p.61-68, 2001.
MERLIM, A.O.; GUERRA, J.G.M.; JUNQUEIRA, R.M.; AQUINO, A.M. Soil macrofauna in cover crops of figs grown under organic management. Scientia Agricola, v.62, p.57-61, 2005.

ODUN, E.P. Ecologia. Rio de Janeiro: Editora Guanabara Koogan, 1988. 639p.

OLIVEIRA, F.H.T.; NOVAIS, R.F.; ALVAREZ V.H.V.; CANTARUTTI, R.B.; BARROS, N.F. Fertilidade do solo no sistema plantio direto. Tópicos em Ciência do Solo, v.2, p.393486, 2002.

OLIVEIRA, J.O.A.P.; VIDIGAL FILHO, P.S.; TORMENA, C.A.; PEQUENA, M.G.; SCAPIM, C.A.; MUNIZ, A.S.; SAGRILO, E. Influência de sistemas de preparo do solo na produtividade da mandioca (Manihot esculenta, Crantz). Revista Brasileira de Ciência do Solo, v.25, p.443-450, 2001.

SILVA, R.F.; AQUINO, A.M.; MERCANTE, F.M.; GUIMARÃES, M.F. Macrofauna invertebrada do solo sob diferentes sistemas de produção em Latossolo da região do cerrado. Pesquisa Agropecuária Brasileira, v.41, p.697-704, 2006a.

SILVA, R.F.; AQUINO, A.M.; MERCANTE, F.M.; GUIMARÃES, M.F. Populações de oligoquetos (Annelida: Oligochaeta) em um Latossolo Vermelho submetido a sistemas de uso de solo. Ciência Rural, v.36, p.673-677, 2006 b.

SOUZA, L.D.; SOUZA, L.S. Manejo do solo para mandioca. In: OTSOBU, A.A.; MERCANTE, F.M.; MARTINS, C.S. (Ed.). Aspectos do cultivo da mandioca em Mato Grosso do Sul. Dourados: Embrapa Agropecuária Oeste; Campo Grande: UNIDERP, 2002. p.109-125.

WOLTERS, V. Invertebrate control of soil organic matter stability. Biology and Fertility of Soil, v.31, p.1-19, 2000.

Recebido em 28 de fevereiro de 2007 e aprovado em 9 de maio de 2007 